\title{
A hospital based Study on Co-morbidities in children with severe acute malnutrition
}

\author{
$\operatorname{Garg} \mathbf{M}^{1}$, Devpura $K^{2}$, Saini S. $K^{3}$, Kumara $S^{4}$ \\ ${ }^{1}$ Dr. Manisha Garg, Senior Resident, ${ }^{2}$ Dr Kusum Devpura, Unit Head and Professor, ${ }^{3}$ Dr. Susheel Kumar Saini, Resident, \\ above all authors are affiliated with Department of Paediatrics, SMS Medical College, Jaipur, India, ${ }^{4}$ Dr Seema kumara, \\ Anesthesia, PGIMER, Rohtak, Haryana, India.
}

Address for Correspondence: Dr. Manisha Garg, House No. 28, M.P. Colony, Mantown, Sawai Madhopur, Rajasthan, India. Email:drgargmanisha@gmail.com

\begin{abstract}
Introduction: Severe acute malnutrition, is characterized by wasting (marasmus), oedema (as a result of kwashiorkor), or both (marasmic kwashiorkor), and occurs mostly in children. Globally, co morbidities such as diarrhoea, acute respiratory tract infections and Malaria, which results from a relatively defective immune status, remain the major causes of death among children with severe acute malnutrition. This study was carried out to find out co-morbidities such as infections and micronutrient deficiencies in children with severe acute malnutrition. Methods: In this hospital based descriptive type of observational study, 125 severe acute malnourished children were included. Patients undergo relevant investigation to find out associated infectious co morbidities. Micronutrient deficiencies assessed by clinical signs. Results: $42 \%$ had diarrhea and $27 \%$ had acute respiratory tract infections as co morbid condition. Tuberculosis was diagnosed in $13 \%$ of cases. Anemia was present in $86 \%$ cases. Signs of vitamin B and vitamin A deficiency were seen in $24 \%$ and $6 \%$ cases. $97 \%$ children have inadequate vitamin D levels. Conclusions: Timely identification and treatment of various co-morbidities is likely to break undernutrition-disease cycle, and to decrease mortality and improve outcome. Nearly all SAM patients have inadequacy of Vitamin D. So Vitamin D supplement should be given to all SAM patients.
\end{abstract}

Keywords: Severe acute malnutrition, Mortality, Under-five children, Co-morbidity

\section{Introduction}

Severe Acute Malnutrition (SAM) is defined as a weight for height measurement of $70 \%$ or less below the median, or three SD or more below the mean National Centre for Health Statistics reference values, the presence of bilateral pitting oedema of nutritional origin, or a mid upper arm circumference of less than $110 \mathrm{~mm}$ in children age 1-5 years [1]. Severe acute malnutrition, is characterized by wasting (marasmus), oedema (as a result of kwashiorkor), or both (marasmic kwashiorkor), and occurs mostly in children [2].

According to the national family health survey prevalence of severe wasting is $7.9 \%$ as per WHO Child Growth Standards [3]. Out of 19 million children suffering from severe acute malnutrition in all developing countries, 8 million are in India, twice than in sub-Saharan Africa. Severe acute malnutrition affects

Manuscript received: $24^{\text {th }}$ December 2016

Reviewed: $30^{\text {th }}$ December 2016

Author Corrected: $7^{\text {th }}$ January 2017

Accepted for Publication: $13^{\text {th }}$ January 2017 an estimated 20 million children under 5 years of age and is associated with 1-2 million preventable child deaths each year [4].

Severe acute malnutrition (SAM) results from a nutritional deficit that is often complicated by marked anorexia and concurrent infective illness [5]. Similarly, malnutrition increases one's susceptibility to and severity of infections and is thus a major component of illness and death from disease. Globally, comorbidities such as diarrhoea, acute respiratory tract infections and Malaria, which results from a relatively defective immune status, remain the major causes of death among children with SAM [6]. Anemia, Vitamin B complex deficiency, Vitamin D deficiency, Vitamin A deficiency, Scurvy are the common micronutrient deficiencies seen in severe acute malnourished Children [7]. This study was carried out to find out co-morbid ities such as infections and micronutrient deficiencies in children with severe acute malnutrition. 


\section{Methods}

This study was conducted in the Department of Pediatric Medicine, Sir Padampat Mother and Child Health Institute, attached to SMS Medical College, Jaipur from May 2014 to April 2015. A total of 125 cases presenting with severe acute malnutrition were enrolled of age 6 to 59 months. Severe acute malnutrition among children of six to fifty nine month of age is defined by WHO and UNICEF as any of the following [8]-

1. Weight for height below -3 standard deviation of median WHO growth reference

2. Mid upper arm circumference below $11.5 \mathrm{~cm}$

3. Presence of bipedal oedema

4. Visible severe wasting

Children whose guardians refuse to give positive consent and those who died before taking necessary investigations were excluded. Children with major congenital malformations and those with chronic systemic diseases such as chronic kidney disease, chronic liver disease were also excluded.

This study was descriptive type of observational study. The clinical and the demographic information were recorded on a pre-structured proforma, together with the detail history, physical and detailed systemic examination.

Weight, length/height, Mid-Upper Arm Circumference (MUAC) and weight for height/ length were determined from each study participant. Socioeconomic status of study subjects was assessed as advised by Modified Kuppuswamy scale of social classification which is based on occupation, education of the parents and income of the family [9]. Contact with tuberculosis was determined by either contact with open case of pulmonary tuberculosis recently or if there is history that the child was in contact of open case of tuberculosis in last two year of time. Immunization status of study subjects was assessed as per schedule of National Immunization Programme (NIP) [10].

Infectious co morbidities defined as per following criteria-

Diarrhoea was defined as three or more loose stools per day for any time duration. Persistent diarrhoea was defined as an episode of diarrhoea, of presumed infectious etiology, which starts acutely but lasts for more than 14 days. Chronic diarrhoea was defined as insidious onset diarrhoea of $>2$ weeks duration in children. Acute respiratory tract infection was defined as short duration of cough $(<2$ weeks) or respiratory difficulty, age-specific fast breathing (above normal for age category), auscultatory and/or chest x-ray findings.

UTI (Urinary tract infection) was diagnosed on the basis of suggestive clinical symptoms along with positive urine culture report. Measles was defined as generalized maculopapular rash lasting for $\geq 3$ days, fever $\left(\geq 38.3^{\circ} \mathrm{C}\right.$, if measured) along with cough, coryza (i.e. runny nose) or conjunctivitis (i.e. red eyes). Meningitis was diagnosed on the basis of suggestive clinical features and confirmed by CSF examination and neuroimaging.

Indian Academic of Peadiatric algorithm was applied to diagnose the tuberculosis in children in this study [11]. Anaemia was defined on the basis of WHO reference values of hemoglobin ( $\mathrm{Hb}$ ) in children in age group of 6 to 59 months [12].

\begin{tabular}{|c|c|}
\hline Anemia & Hb level (gm/dl) \\
\hline Mild & $10-10.9$ \\
Moderate & $7-9.9$ \\
Severe & $<7$ \\
\hline
\end{tabular}

Micronutrient deficiencies were assessed by clinical signs during general physical examination in these children except Vitamin D status which was determined by laboratory test. Vitamin A deficiency defined clinically by presence of night blindness, Bitot's spots, corneal xerosis and/ or ulcerations, corneal scars caused by keratomalacia. Vitamin B complex deficiency defined clinically by presence of angular stomatitis, cheilosis, glossitis, dermatitis, tingling/numbness in the extremities. Scurvy defined clinically by gum bleeding, loose teeth, Joint pains, Dry scaly skin, delayed wound-healing with suggestive findings of X-ray long bones. 
Vitamin D status (25 Hydroxy Vitamin D) in study subjects was determined by laboratory test by chemiluminescence method using ADIVA CENTOR XP machine. Vitamin D deficiency is defined as serum levels of 25(OH)D less than 20 $\mathrm{ng} / \mathrm{dL}$ whereas 21- $29 \mathrm{ng} / \mathrm{dL}$ is considered to be insufficient [13].

A written, informed consent was obtained from parents. Clearance from Departmental Ethics Committee was taken prior to the start of the study. All participants had the option to withdraw from the study anytime during their hospital stay.

All filled questionnaires were checked and coded on Microsoft Office Excel Worksheet and any missing data or information was actively searched from patient's files. Descriptive cross tabulations were formed to examine for associations.

\section{Results}

Baseline characteristics- In this study, 125 children with severe acute malnutrition were included. The mean age of presentation was 20.4 months. Among study population $35(28 \%)$ children were of age group $6-12$ month. $66(52.8 \%)$ children related to age group 13 - 24 month while 24(19.2\%) children belonged to age group of $25-59$ month. Among the children $49(39 \%)$ were female while $76(61 \%)$ were male. Ratio of male to female patients was 1.55:1.

Most of the children were belonged to lower socio economic class. 87 (70\%) children belonged to upper lower class, 35 ( $28 \%$ ) belonged to lower middle class while $3(2 \%)$ children belonged to upper middle class. $83(66 \%)$ children had the history of recurrent hospitalization; either by same illness or other illness.

Among the cases, 107(86\%) had weight for height <- 3SD, 50(40\%) children had visible severe wasting, 102(82\%) had mid upper arm circumference $<11.5 \mathrm{~cm}$, while $20(16 \%)$ had bilateral pitting oedema of nutritional origin.

Among the cases, 30 (24\%) were completely immunized, 82 (65\%) were partially immunized while 13 (10\%) were unimmunized. 95 (76\%) children were found to receive exclusive breast feeding till 6 month of age. While complimentary feeding started in only $31(25 \%)$ children at 6 month of age. History of contact with tuberculosis was present in 17 (14\%) children. (Table no.1)

Clinical data- The most common presenting complaint which was seen in current study was fever. That was present in 93 (74.4\%) of cases. Other more common presenting complaints in study subjects were loose motion in 55(44\%), cough in 51 $(40.8 \%)$, decreased oral acceptance in 32 (25.6\%), vomiting in $28(22.4 \%) .25(20 \%)$ children presented with Respiratory distress. Other less common presenting complaints were irritability in $19(15.2 \%)$ children, swelling over body either localized or generalized in $17(13.6 \%)$ children, Seizures of any type in $11(8.8 \%)$ children and rashes over body in $6(4.8 \%)$ children. (Table no.2)

Co-morbidity Data- Diarrhoea was found to be most common infectious co-morbidity. That was present in 53 (42\%) of children. Among 53 cases of diarrhoea, 38 (71.6\%) had acute diarrhoea, $9(16.9 \%)$ had chronic diarrhoea while $6(11.3 \%)$ had persistent diarrhoea. Acute respiratory tract infections were second most common co-morbidity which was seen in 34 $(27 \%)$ children.16 (13\%) children had tuberculosis as co-morbid condition. Out of 16 children 9 had pulmonary tuberculosis while 7 children had tubercular meningitis. Urinary Tract Infection

(UTI) was diagnosed in 11(9\%) children. Out of 11 children diagnosed to have UTI; 4 children had growth of Escherichia coli, 3 children had Candida while other had coagulase negative staphylococci (CONS), coagulase positive staphylococci (COPS), Enterobacter and Pseudomonas (one case each). Measles was seen in $6(5 \%)$ children. Pyomeningitis was diagnosed in 5 (4\%) children. (Table no.3)

Among the study subjects 108 (86\%) children were found anemic. Out of 108 anemic patients; 17 (15.7\%) children had mild anemia, 54 (50\%) had moderate anemia while 37 (34.2\%) children had severe anemia.

Vitamin A deficiency was present in 6 (5\%) children. Vitamin B complex deficiency seen in 24 (19\%) children while scurvy seen in $2(1.6 \%)$ of children. Inadequate levels of Vitamin D were present in 121 (97\%) children. (Table no.4) 
Table No.-1: Baseline characteristics of study subjects.

\begin{tabular}{|l|c|}
\hline Patients, No. (\%) & $\mathbf{1 2 5}(\mathbf{1 0 0 \% )}$ \\
\hline Age group, No. (\%) & $35(28 \%)$ \\
- $6-12$ month & $66(52.8 \%)$ \\
- $13-24$ month & $24(19.2 \%)$ \\
- $25-59$ month & \\
\hline Sex, No. (\%) & $49(39 \%)$ \\
- Female & $76(61 \%)$ \\
- Male & $1: 1.6$ \\
- F: M ratio & \\
\hline Immunization status & $30(24 \%)$ \\
- Completely immunized & $82(65 \%)$ \\
- Partially immunized & $13(10 \%)$ \\
- Not immunized & $87(70 \%)$ \\
\hline Socioeconomic status & $35(28 \%)$ \\
- Upper lower & $3(2 \%)$ \\
- Lower middle & $83(66 \%)$ \\
- Upper middle & $95(76 \%)$ \\
\hline History of recurrent hospitalization & $31(25 \%)$ \\
\hline Exclusive breastfeed till 6 month of age & $17(14 \%)$ \\
\hline Complimentary feeding started at 6 month of age & \\
\hline History of contact with tuberculosis & \\
\hline
\end{tabular}

Table No.-2: Presenting complaints in study subjects-

\begin{tabular}{|l|c|}
\hline Patient No. (\%) & $125(100 \%)$ \\
\hline Fever & $93(74.4 \%)$ \\
\hline Loose Motion & $55(44 \%)$ \\
\hline Cough & $51(40.8 \%)$ \\
\hline Decreased oral acceptance & $32(25.6 \%)$ \\
\hline Vomiting & $28(22.4 \%)$ \\
\hline Respiratory distress & $25(20 \%)$ \\
\hline Irritability & $19(15.2 \%)$ \\
\hline Swelling & $17(13.6 \%)$ \\
\hline Seizure & $11(8.8 \%)$ \\
\hline Rash & $6(4.8 \%)$ \\
\hline
\end{tabular}

Table No.-3: Distribution of cases according to their infectious co-morbidity-

\begin{tabular}{|l|c|}
\hline Patient No. (\%) & $\mathbf{1 2 5 ( 1 0 0 \% )}$ \\
\hline Diarrhoea & $53(42 \%)$ \\
\hline Acute respiratory infection & $34(27 \%)$ \\
\hline Tuberculosis & $16(13 \%)$ \\
\hline Urinary tract infection & $11(9 \%)$ \\
\hline Measles & $6(5 \%)$ \\
\hline Pyogenic meningitis & $5(4 \%)$ \\
\hline
\end{tabular}


Table No.-4: Micronutrient deficiencies in study subjects-

\begin{tabular}{|l|c|}
\hline Patients, No. (\%) & $125(100 \%)$ \\
\hline Anemia & $108(86 \%)$ \\
Mild & $17(15.7 \%)$ \\
Moderate & $54(50 \%)$ \\
Severe & $37(34 \%)$ \\
\hline Vitamin A deficiency & $6(5 \%)$ \\
\hline Vitamin B complex deficiency & $24(19 \%)$ \\
\hline Scurvy & $2(1.6 \%)$ \\
\hline Vitamin D status & $121(97 \%)$ \\
Deficient & $42(34 \%)$ \\
Insufficient & $79(63 \%)$ \\
Adequate & $4(3 \%)$ \\
\hline
\end{tabular}

\section{Discussion}

Severe acute malnutrition is a well recognized emergency situation with substantial morbidity and mortality that requires immediate and effective treatment. Prompt initiation of appropriate treatment of both complications such as hypoglycemia, hypothermia, dehydration, electrolyte imbalance, infections and the underlying cause can significantly reduce associated morbidity and mortality.

Most of the studies are related to infectious comorbidities while only few studies are related to micronutrient deficiencies in severely malnourished children. Mean age of children reporting with malnutrition was similar to other studies and there was significant male predominance in malnourished children [14].

This may be due to ignorance about the health checkups of female children. However, no definite causal relationship was found for this male preponderance. Our study show less coverage of immunization in our study area which is likely due to ignorance and unawareness about immunization.

In our study about two third children had the history of recurrent hospitalization; either by same illness or other illness. In study by Yoann Madec el al history of recurrent hospitalization was present in $4.7 \%$ children only [15]. Unhygienic living conditions and poor socioeconomic status may be the cause of increased rate of hospitalization in our study subjects. Rate of exclusive breast feeding found to be more in our study but weaning not started at recommended age which is predisposing factor for malnutrition. In present study, Diarrhoea was found to be the most common infectious co-morbidity. Acute respiratory tract infections were found to be the second most common co-morbidity followed closely by tuberculosis. In a Colombian study, $68.4 \%$ of malnourished children were suffering from diarrhea and $9 \%$ had sepsis at the time of admission [16].

Two African studies also showed high incidence of diarrhea in SAM children of $49 \%$ and $67 \%$ $[17,18]$.Though previous reports have described malnutrition as an important risk factor for pneumonia than for diarrhea[19], diarrhea was the major co-morbid condition found in our study. A study from Africa also reported a comparable incidence of respiratory illness and tuberculosis (18\% each) in admitted SAM Children [20]. Measles has severe consequences on the nutritional status. A previous Indian study [8] showed only 3.8\% of children with past history of measles but we found a higher proportion. Malaria and HIV infection were previously reported as major co-morbidities with total prevalence of $21 \%$ and $29.2 \%$, respectively[21] but In our study malaria, HIV were not found to be as comorbid condition.

Overlapping nature of protein-energy malnutrition and micronutrient deficiencies is well understood and it is seen that lack of one micronutrient is typically associated with deficiency of others[22]. Anemia and vitamin D deficiency were the two most common micronutrient deficiencies associated with malnutrition in our study, and this is consistent with the previous reports[23]. 
In previous studies vitamin $\mathrm{D}$ status is accessed by clinical examination and radiological findings but in our study vitamin D status is accessed by laboratory test. So we found higher incidence of Vitamin D deficiency. Absence of a comparative group, no biochemical evaluation for micronutrient deficiencies and nonassessment of contributing factors for these deficiencies were the main lacunae of the study.

Our institute is the only referral centre and the major hospital that provides primary to tertiary care for children in this region. The geographic location of our hospital may have led to an enrollment bias.

Our study suggest that malnutrition is predicted by age less than two years, failure to start complimentary feeding at recommended age, recurrent hospitalization, taking unbalanced diet, lack or incomplete immunization and lower socioeconomic status.

\section{Conclusion}

Apart from nutritional rehabilitation, timely identification and treatment of co-morbidities like diarrhea, acute respiratory tract infection, anemia and micronutrient deficiencies is vital in malnourished children, so as to break undernutrition-disease cycle, and to decrease mortality and to improve outcome.

Nearly all SAM patients have inadequacy of Vitamin D. So Vitamin D supplement need to be given to all SAM patients.

Funding: Nil, Conflict of interest: None initiated, Perission from IRB: Yes

\section{References}

1. Collins S, Dent N, Binns P, Bahwere P, Sadler K, Hallam A. Management of severe acute malnutrition in children. Lancet. 2006 Dec 2;368(9551):1992-2000.

2. Bhan MK, Bhandari N, Bahl R. Management of the severely malnourished child: perspective from developing countries. BMJ. 2003 Jan 18; 326 (7381):146-51

3. International Institute for Population Sciences (IIPS) and Macro International. National Family Health Survey (NFHS-3), 2005-2006. Mumbai: IIPS; 2007.

4. World Health Organization (WHO)- Guidelines for the inpatient treatment of severely malnourished children. Geneva: WHo; 2003.
5. Manary MJ, Sandige HL. Management of acute moderate and severe childhood malnutrition. BMJ. 2008 Nov 13;337:a2180. doi: 10.1136/bmj.a2180.

6. Black RE, Cousens S, Johnson HL, Lawn JE, Rudan I, Bassani DG, Jha P, Campbell H, Walker CF, Cibulskis R, Eisele T, Liu L, Mathers C; Child Health Epidemiology Reference Group of WHO and UNICEF. Global, regional, and national causes of child mortality in 2008: a systematic analysis. Lancet. 2010 Jun 5; 375 (9730):1969-87. doi: 10.1016/S0140-6736(10) 60549-1. Epub 2010 May 11.

7. Rakesh kumar, Jyoti singh, Karan joshi, H P singh and $\mathrm{S}$ bij. Co-morbidities in Hospitalized Children with Severe Acute Malnutrition, August 05, 2013. PII: S097475591300461.

8. World Health Organization. Management of Severe Malnutrition: A Manual for Physicians and Other Senior Health Workers, World Health Organization, Zeneva, Switzerland, 1998.

9. BP Ravi Kumar, Kuppuswamy's Socio-Economic Status Scale - a revision of economic parameter for 2012, International Journal of Research \& Development of Health. Jan 2013; Vol 1(1): 2-4.

10. Vinod k Paul, Arvind Bagga et al.,. Essential pediatrics, National immunization programme, page 206, ISBN 978-81-239-2334-5.

11. Working Group on Tuberculosis, Indian Academy of Pediatrics (IAP). Consensus statement on childhood tuberculosis. Indian Pediatr. 2010 Jan;47(1):41-55.

12. World Health Organization: WHO, UNICEF, and SCN informal consultation on community-based management of severe malnutrition in children, SCN Policy Paper No. 21. Geneva: World Health Organization; 2006.

13. Holick MF, Binkley NC, Bischoff-Ferrari HA, Gordon CM, Hanley DA, Heaney RP, Murad MH, Weaver CM; Endocrine Society. Evaluation, treatment, and prevention of vitamin D deficiency: an Endocrine Society clinical practice guideline. J Clin Endocrinol Metab. 2011 Jul; 96(7):1911-30. doi: 10.1210/jc.20110385. Epub 2011 Jun 6.

14. Bachou H, Tylleskär T, Deogratias H, Mulindwa K,Tumwine JK. Bacteraemia among severely 
malnourished children infected and uninfected with the Human immunodeficiency virus-1 in Kampala, Uganda. BMCInfect Dis. 2006; 6:160.

15. Madec Y, Germanaud D, Moya-Alvarez V, Alkassoum W, Issa A, Amadou M, Tchiombiano S, Pizzocolo C, Huber F, Diallo S, Abdoulaye-Mamadou R. HIV prevalence and impact on renutrition in children hospitalised for severe malnutrition in Niger: an argument for more systematic screening. PLoS One. 2011; 6(7):e22787. doi: 10.1371/journal.pone.0022787. Epub 2011 Jul 28.

16. Bernal C, Velásquez $\mathrm{C}$, Alcaraz $\mathrm{G}$, Botero J. Treatment of severe malnutrition in children: experience in implementing the World Health Organization guidelines in Turbo, Colombia. J Pediatr Gastroenterol Nutr. 2008 Mar; 46(3): 322-8. doi: 10. 1097/MPG.0b013e318156c2c3.

17. Talbert A, Thuo N, Karisa J, Chesaro C, Ohuma E, Ignas J, Berkley JA, Toromo C, Atkinson S, Maitland K. Diarrhoea complicating severe acute malnutrition in Kenyan children: a prospective descriptive study of risk factors and outcome. PLoS One. 2012;7(6):e38321. doi: 10.1371/journal.pone.0038321. Epub 2012 Jun 4.
18. Irena AH, Mwambazi M, Mulenga V. Diarrhea is a major killer of children with severe acute malnutrition admitted to inpatient set-up in Lusaka, Zambia. Nutr J. 2011 Oct 11;10:110. doi: 10.1186/1475-2891-10-110.

19. Berkowitz FE. Infections in children with severe protein-energy malnutrition. Pediatr Infect Dis J. 1992 Sep;11(9):750-9.

20. Sunguya BF, Koola JI, Atkinson S. Infections associated with severe malnutrition among hospitalised children in East Africa. Tanzan Health Res Bull. 2006 Sep; 8(3):189-92.

21. Sunguya B. Effects of Infections on Severely Malnourished Children in Kilifi-Mombasa and Dar es Salam : A Comprehensive Study. Dar es salaam Medical Students Journal. 2006;14(1):27-35.

22. Bhaskaram P. Measles and malnutrition. Indian $\mathbf{J}$ Med Res. 1995;102:195-99.

23. Ejaz MS, Latif N. Stunting and micronutrient deficiencies in malnourished children. J Pak Med Assoc. $2010 \mathrm{Jul} ; 60$ (7):543-7.

\section{How to cite this article?}

Garg M, Devpura K, Saini S. K, Kumara S. A hospital based Study on Co-morbidities in children with severe acute malnutrition. J PediatrRes.2017;4(01):82-88.doi:10.17511/ijpr.2017.i01.16 